\title{
Mon sida de Jean-Paul Aron : l'autocensure ou le jugement du miroir
}

\section{JoRge CALderon}

Etudiant au doctorat au Departement de langue et littérature françaises de l'Université McGill, Jorge Calderon (jcalderon11@hotmail.com) s'interesse à Jean-Paul Sartre, Simone de Beauvoir, le Nouveau Roman et les érivains du sida. Son article est la mise en lumière et l'interpretation du premier temoignage public d'un intellectuel français sur le sida et il se veut aussi une contribution à la critique et à la théorie du texte gay.

La peur de la différence est inhérente à nos sociétés. Nul ne peut revendiquer son identité sans mettre en péril le fragile équilibre de la structure sociale que l'autre a construite. Ce fut le cas pour les mouvements ouvriers, les groupes de libération des noirs en Amérique et en Afrique, la vague féministe. Ce qui est intéressant, c'est de remarquer que ces luttes se sont inscrites aussi bien dans la réalité concrète que sur le papier. Pensons simplement à tous ces manifestes, ces romans, ces poèmes, ces pièces de théâtre, qui revendiquaient une nouvelle interprétation de la vérité sociale, une remise en question des valeurs religieuses et nationalistes, une relecture des différences. Ces prises de parole ont été sévèrement critiquées : par exemple le blâme et la condamnation a été le lot, pendant longtemps, de l'œuvre de Simone de Beauvoir. Sans aucun examen préalable, certains condamnaient toute production émanant de ces nouvelles formes de pensée : le pouvoir logocentrique ne 
tolérant aucune œuvre, aucun spectacle, aucune prise de position et de parole, qui le remette en question - rien n'est pensable, rien n'est dicible, rien n'est scriptible, hors de lui-même. Néanmoins, certaines personnes osent parler et écrire à partir des marges et ainsi elles redéfinissent le centre. Pourtant ce n'est pas parce que j'ai le droit de parler que j'ai la possibilité de le faire - ou au moins de le faire conformément à mon intention première. Celui qui prend la parole ne se trouve plus face à la censure de l'autre, mais bien face à luimême : il s'autocensure, il se condamne au silence ou bien à une certaine distorsion de son propos. En se privant de sa liberté, le sujet exerce la plus terrible des censures : celle qui interdit à soi-même de penser, de dire et d'écrire sa vérité. Ce phénomène est lisible dans les textes gays et lesbiens. Il n'est pas nécessaire de rappeler l'opprobre que les préférences sexuelles ont soulevé et soulèvent toujours. Dans ce contexte social revendiquer une identité marginale n'est pas un acte simple, et les meilleures intentions n'y peuvent rien. Se dire l'idem de celui qui est stigmatisé n'est pas facile. Accepter le caractère de l'identité gaie et lesbienne comme une unité ne va pas de soi. Ce sont ces problèmes de relationnement entre une identité personnelle et une identité culturelle qui sont difficiles à formuler et à envisager. Ainsi, accepter qu'on appartient à un groupe marginal et marginalisé - parce que minoritaire -, accepter de reconnaître un ensemble de traits propres à la communauté des bannis et s'y reconnaître soi-même n'est pas chose facile. Il y a donc pour moi un refoulement évident dans l'inconscient du texte gay et lesbien des éléments de la pensée de soi-même et du désir de se dire en s'identifiant au groupe auquel on appartient - sans avoir voulu en être. Celui qui parle ou écrit se retrouve ainsi face à une société, ou plutôt à l'image qu'il connaît de celle-ci, qui lui est hostile et qui ne tolère d'aucune manière ce qu'il à dire, ce qu'il a à lui dire. Et ce n'est pas parce qu'à un premier degré de sens on fait l'apologie, l'éloge et la louange de son identité (culturelle), qu'à un deuxième degré de sens on ne sent pas le poids de la faute qu'on croit être en train de commettre. C'est à partir de ces données, la prise de parole, l'homosexualité, le sida et la censure/autocensure, que j'analyserai le texte de Jean-Paul Aron, Mon sida. 
Avant de passer à l'analyse textuelle en tant que telle, j'aimerais rappeler le contexte socio-historique qui a vu éclore la littérature du sida. Ainsi, depuis 1981, la société occidentale, et plus particulièrement la communauté homosexuelle, a été confrontée à l'apparition d'un nouveau fléau : le sida. Une importante documentation en sciences de la santé, sociales et humaines a été produite afin de mieux comprendre le phénomène et dans l'espoir d'arrêter la progression du sida ou du moins de réduire sa vitesse de propagation. Il est indéniable que le sida a créé une nouvelle division sociale entre les séro-négatifs et les séro-positifs, simples porteurs du virus ou personnes gravement atteintes des suites du développement du syndrome. Appartenir au second groupe est une calamité pour plusieurs raisons : le sida est une condamnation à une mort précoce et douloureuse, l'individu se trouve du jour au lendemain face à une réalité qui le force à réévaluer son identité et son mode de vie, et surtout le séro-positif est la victime d'une angoisse sociale qui le marginalise doublement en faisant peser sur lui le soupçon de l'homosexualité et la peur de la contamination dont il serait la source. Le sida, après une période relative de "Révolution sexuelle", qu'on peut d'ores et déjà juger comme ayant été timide et superficielle, réveille les pires tabous sociaux à l'encontre de ce que les médecins et les sociologues ont appelé "le groupe à risque" : les homosexuels. Ce groupe a été des plus touchés et des plus stigmatisés par le sida. L'homosexualité est redevenue le signe d'une maladie se présentant comme une épidémie, comme un danger de perturbation sociale et de mort. Cependant, ce que des journalistes ont appelé au tout début "le cancer des homosexuels" est rapidement devenu "une peste fin de siècle" touchant toutes les couches de la société, aussi bien les "homos" que les "hétéros". Même si les données ont changé, il n'en reste pas moins que le sida est largement associé à des pratiques sexuelles marginales et clandestines, ainsi qu'à la toxicomanie. Ajoutons que les préjugés sociaux ont bénéficié du symbolisme de deux vecteurs principaux dans la transmission du sida : le sang et le sperme - ayant tous les deux de lourdes connotations de "pureté" et de "faute". Et même si aujourd'hui, contrairement au début des années 80 , la médecine a démontré que le virus est uniquement transmis par des vecteurs biologiques, certaines personnes continuent de croire que des facteurs 
psychologiques, spirituels et autres balivernes entrent dans la contamination par le sida. Comme le rappelle Michael Pollak : "Cette confusion a donc créé chez beaucoup d'homosexuels le sentiment qu'ils étaient dénoncés moins pour ce qu'ils faisaient que pour ce qu'ils étaient" (12). Pour ces personnes, ce ne sont pas les pratiques sexuelles à risque qui sont mises en cause, mais uniquement la préférence sexuelle. Les tenants de cette pensée magique prétendent encore aujourd'hui que les hétérosexuels ne sont pas à risque. Ainsi, le discours sur le sida est devenu un révélateur des valeurs, des préjugés, de la morale et avant tout des tensions qui existent dans notre société. Il a aussi été un catalyseur de l'identité gaie et lesbienne - termes dénotant l'existence d'un groupe social culturel sans connoter une relation univoque, directe et réductrice à la sexualité.

Dans un tel contexte se dire gay et sidatique devenait très problématique. Le premier intellectuel français à avoir avoué publiquement sa maladie est Jean-Paul Aron. Mon sida a été un témoignage choc qui s'est inscrit dans l'horizon du discours social de l'époque. Dans ce texte, le dévoilement de l'auteur est aussi une révélation (voilée) d'une profonde culpabilité. Jean-Paul Aron se dit homosexuel et fier de l'être. Toutefois son discours révèle autre chose - je rappelle la notion d'intentionnalité : la langue dit toujours quelque chose, et ce qu'elle dit ne correspond pas forcément à l'intention manifeste de celui qui parle et/ou écrit. Dans mon analyse je m'efforcerai de montrer l'enjeu d'une herméneutique de la révélation - en prenant la parole, le témoin du sida se révèle autrement à lui-même tout en nous révélant une expérience nouvelle - qui instaure une subtile relation entre une poétique de l'implicite et de l'explicite. À mon avis, le témoignage de Jean-Paul Aron est une auto-justification fonctionnant sur un profond sentiment de culpabilité : tout d'abord parce qu'il doit avouer une homosexualité problématique et puis, bien sûr, parce qu'il est atteint du sida. Son discours fonctionne sur le mode de l'aveu, mais aussi de la délation : l'auteur rappelle que Michel Foucault est mort du sida. En révélant le silence, et par conséquent une prétendue faute éthique concomitante au sida, Jean-Paul Aron fait œuvre de transparence tout en instaurant une problématique complexe du dit et du non-dit. 


\section{Un témoignage sous forme d'entretien}

Mon sida, publié en 1988 aux éditions Christian Bourgois, est paru tout d'abord dans le Nouvel Observateur du 30 octobre et du 5 novembre 1987. Les propos de Jean-Paul Aron ont été recueillis par Élisabeth Schemla. Aussi, le témoignage prend la forme d'un entretien. Le texte est donc à deux voix . C'est la deuxième voix qui commence le texte en restituant l'apparence physique de l'auteur : "Très amaigri, sans que sa silhouette ait perdu de son élégance un peu voûtée" (7); sa voix : "régulièrement interrompu[e] par une toux sèche ou un bâillement de fatigue" (7) ; mais aussi la qualité du commentaire et de l'esprit : "le cours implacable d'un propos maîtrisé" (7). La voix d'Élisabeth Schemla est donc le prologue du texte, mais aussi le contrepoint. Dans ce premier passage, Jean-Paul Aron est là : vieilli, malade, parlant avec une voix entrecoupée, mais présent. Et comme s'il fallait toujours être deux pour mieux se comprendre, elle écrit : "son regard d'urgence ne lâche pas une seconde le regard qui lui fait face, comme s'il était à l'affût du reflet enfin fidèle de son être" (7). Ce passage est à mettre en rapport avec la pensée de Mikhaïl Bakhtine qui, dans un tout autre contexte, écrivait qu'une voix seule n'est et ne peut rien : “Tout n'est que moyen, le dialogue est le but. Une voix seule ne finit rien, ne résout rien. Deux voix sont un minimum de vie, d'existence" (344). Ainsi, soi n'existe pas sans/hors relation, car il se définit et il est défini par ce qui est autre. Ce qui est important, c'est de plonger la pensée dans une relativité de l'existence en devenir et de ne pas la laisser se figer en un raidissement abstrait et dogmatique. Ce qui compte avant tout, c'est l'angle dialogique sous lequel tout s'oppose ou se juxtapose dans le texte. Pour Bakhtine, le dialogue est la seule vérité intrinsèque de tout discours. Par conséquent, il me semble que ce premier témoignage public d'un intellectuel français atteint du sida marque bien la nécessité de son existence par la forme choisie : l'entretien. Jean-Paul Aron se place immédiatement en situation de communication directe par le dialogue. Ses propos, même s'ils ont été profondément médités, sont rapidement reçus par son interlocutrice et ils sont par la suite rapidement publiés dans un journal à grand tirage. Touchant par le fait même un large public, et ce dans un délai plutôt bref. Le premier but du texte est donc d'informer le public en démystifiant la réalité du sida. La forme du 


\section{2 / Calderon}

texte et son lieu de publication témoignent donc de l'urgence du propos transmis. Il y a plus : le discours de Jean-Paul Aron est aussi une quête ontologique qui ne peut avoir lieu qu'à travers, par et dans le langage. Ce n'est que par l'expression de ce qu'il dit que l'auteur prend conscience de ce qu'il est. Ce discours se veut un miroir fidèle. Toutefois, le miroir révèle une image beaucoup plus vaste que ce que Jean-Paul Aron aurait peut-être voulu laisser voir : le miroir a deux faces, et l'une peut refléter l'autre à l'infini.

\section{Auto-justification et culpabilité}

Cette remarque nous amène à notre deuxième point : le discours de l'auteur se veut le témoignage privilégié d'un intellectuel français sur une situation nouvelle à travers son expérience vécue. Il se voudrait donc l'illustration et la démystification du sida. Toutefois, le discours véhicule un autre sens : il prend aussi la forme d'une auto-justification. Ainsi, parler dans un entretien à l'autre, c'est parfois, sinon souvent, se parler à soi-même à haute voix. Justifier la condition d'un groupe stigmatisé, c'est aussi auto-justifier son propre état. Jean-Paul Aron dit : "Soudainement, une certaine façon de tourner autour du pot, le silence en somme, s'est mise à me gêner" (8). Il y a eu silence de la part de l'auteur autour du sida dans sa vie publique, ainsi que dans sa vie privée et jusque dans sa profonde intériorité. Pourtant, être porteur du sida n'a pas été une information suffisante pour justifier une nouvelle perception de son rapport à lui-même et aux autres. Il a fallu une pneumocystose qui l'a emmené très près de la mort pour que Jean-Paul Aron arrête de "tourner autour du pot" et qu'il prenne conscience de sa nouvelle réalité. Les signes évidents de la dégradation de son état ne lui permettaient plus de garder le silence pour lui-même et pour les autres : il était atteint du sida, une maladie mortelle, et aucun médicament, aucun vaccin ne viendrait à sa rescousse. Cette conscience lui donne l'occasion de dire : "je ne vois pas pourquoi je continuerais à me dissimuler. Notre rencontre et la mise au point de ce texte, au fond, me permettent d'avancer sur la voie d'une partielle libération de moi-même" (9). Mon sida devient donc l'aveu de sa double dissimulation : l'homosexualité et la maladie. Une autre question se pose : quelle est la relation que l'auteur voit entre son orientation sexuelle et le sida? Tout me permet de croire que Jean- 
Paul Aron voyait son homosexualité comme une faute ; et le sida comme un châtiment - même s'il prétend le contraire. Premièrement, il essaie de s'identifier avant tout au dandysme qui dénote une élégance, un raffinement, une attitude morale tout en connotant un détachement face aux valeurs dominantes dans la société et un esthétisme non conformiste. L'auteur le définit quant à lui comme "la différence absolue" (9). Qui dit différence dit souvent marginalité, clandestinité et dissimulation - quand cela est possible. Rappelons que Jean-Paul Aron a donné dès le début des années 80 des interviews à des journaux publiant officiellement pour un public gay et il a participé à des manifestations. Cependant, il parlait de l'homosexualité de manière indirecte, ou du moins il ne parlait pas explicitement de son expérience : “C'est à cette époque, vers 1982-1983, que je me suis montré dans des manifestations d'homosexuels pour défendre leurs droits et leurs libertés. Je savais qu'on prendrait des photos de moi : j'acceptais cet aveu implicite" (15). Je souligne l'utilisation de "leurs droits et leurs libertés" qui exclut du groupe visé celui qui parle. Et ce qu'il accepte, c'est un aveu implicite de son homosexualité. "Aveu", comme il le qualifie, qui peut être interprété comme l'ouverture d'un homme hétérosexuel à la situation des homosexuels marginalisés. L'auteur continue à réfléchir à son rapport à l'homosexualité : “C'était sans doute folie, peut-être inauthenticité, mais je ne me suis jamais senti homosexuel. $\mathrm{La}$ maladie seule m'oblige à convenir que j'appartiens existentiellement et socialement à cette catégorie. J'ai nié ma spécificité, non parce qu'elle me répugnait mais parce que je n'avais pas le désir d' 'en' être" (28). Dans ce passage, il y a une ambivalence qui vaut le commentaire. Tout d'abord, il pose la question d'être homosexuel et de se sentir homosexuel. C'est tout un rapport à la perception de son identité qui se dessine : suis-je ce que je suis sans le percevoir? ou bien suis-je seulement ce que je crois être ? Puis, il déplace la question vers l'existentialisme : l'individu se définit par ses actes et non par ce qu'il est avant même d'exister concrètement. Nous voyons rapidement des contradictions dans ce passage : premièrement Jean-Paul Aron a eu des relations sexuelles avec des hommes, mais il ne se sentait pas homosexuel. Pour un individu qui veut se définir en fonction de ses actes, il y a là un aveuglement. Cependant, ses actes sexuels ont eu comme 
conséquence sa contamination par le VIH. Par la suite le syndrome s'est développé et il a dû être hospitalisé. C'est à ce moment qu'il se définit enfin comme homosexuel, appartenant donc au "groupe à risque". Un peu comme le bouton qui dévoile la peste, le sida a obligé l'auteur à dévoiler et à se dévoiler son homosexualité. Désirant les hommes, il n'avait pourtant pas envie de se cataloguer dans le groupe communément étiqueté "Homosexuels". Il reniait donc ce qu'il était, afin d'éviter, non pas d'être socialement marginalisé, mais bien de s'auto-marginaliser. C'est pour cette raison que je parle d'une profonde culpabilité qui habite l'autojustification de Jean-Paul Aron.

\section{Aveu et délation}

Cette auto-justification prend la forme d'un aveu public. Mais, elle n'est pas seulement un aveu de soi, c'est aussi une forme de délation. Jean-Paul Aron rappelle qu'il a écrit un ouvrage, les Modernes, dont il relisait les épreuves quand Michel Foucault est mort. Dans ce livre, il démolissait les théories et les structures proposées par le philosophe. Il continue : "Foucault était l'homme du langage, du savoir et de la vérité, point du vécu et du sens. Il était aussi homosexuel. Il en avait honte, tout en le vivant parfois de façon insensée. Son silence devant la maladie m'a indisposé parce que c'était un silence de honte, pas un silence d'intellectuel. C'était tellement contraire à tout ce qu'il avait défendu ! Ça m'a paru ridicule !" (27). La première question qui me vient à l'esprit en lisant ce passage est la suivante : de qui parle Jean-Paul Aron? de qui est-il entrain de commenter le comportement? J'aurais tendance à croire que la figure de Michel Foucault devient un miroir herméneutique qui renvoie son propre discours à celui qui parle. Le grand philosophe aurait caché et son homosexualité et sa maladie parce qu'il avait honte de son expérience vécue et du sens que ce vécu aurait produit. Lui, l'homme du langage, du savoir et de la vérité, aurait dissimulé sa situation par honte. (Remarquons que le terme "honte" revient dans deux phrases, l'une à la suite de l'autre.) Sans vouloir disculper Michel Foucault, il faut préciser sa situation en 1984. Le sida était apparu sur la scène publique depuis moins de trois ans. Michel Foucault se sachant atteint n'avait pas à l'époque les informations que Jean-Paul Aron connaissait en 1987. Sans 
oublier que le philosophe avait informé son entourage immédiat de sa nouvelle condition, comme l'a affirmé Hervé Guibert dans des entrevues et dans son best-seller À l'ami qui ne m'a pas sauvé la vie. De plus, comme l'indique Michael Pollak en 1988 : "Les caractéristiques [sociologiques] principales de l'expérience de la maladie sont le secret et le silence, et le maintien dans la mesure du possible d'une continuité de la vie : tout change dans la vision que le malade a de lui-même, mais rien ne doit changer dans l'image que les autres ont de lui" (104-05). Cette inadéquation entre la perception que le malade a de lui-même et celle qu'il souhaite que les autres continuent d'avoir de sa personne est un facteur inhérent à ceux qui sont atteints du sida. Cette maladie instaure une relation tragique entre l'individu et la société, relation fondée sur le non-dit. Le silence devient un bouclier qui permet au malade d'éviter le jugement et le rejet - ce qui serait déjà une première mort sociale de la personne. Cependant, le sidatique ne peut pas dissimuler sa condition très longtemps : dès que les signes physiques apparaissent, la présence du sida est indéniable. Et dans le cas de Michel Foucault, fils d'une famille bourgeoise de province, il faut souligner, comme l'écrit si bien Michael Pollak, que "parfois s'installe une complicité bienveillante entre l'homosexuel et ses proches qui, tout en lui évitant de s'auto-justifier, le protègent contre l'extérieur par un mur du silence" (111). Et j'ajoute que cette situation peut se prolonger au-delà de la mort du malade.

\section{Implicite et explicite}

Après cette mise au point, je veux montrer que Jean-Paul Aron en jugeant Michel Foucault se juge lui-même. Par la délation de l'homosexualité et de la maladie du philosophe, l'auteur dénonce sa propre dissimulation et ses mensonges passés. Ainsi, il nous apprend que son frère aîné et sa mère étaient au courant de son orientation sexuelle. Qu'en est-il de son père ? Quant à la honte, comment celui qui ne voulait pas "en être" peut-il condamner un homme qui a vécu intensément, comme le rapportent ses proches. Quant aux prétendues contradictions entre les théories de Michel Foucault et ses pratiques sociales, Jean-Paul Aron lui-même n'arrête pas de se contredire dans Mon sida. Les médisances de l'auteur n'auraient aucun intérêt si elles ne nous apprenaient pas quelque chose qui peut 


\section{$56 /$ Calderon}

peut-être servir à l'élaboration d'une théorie du texte gay : cet effet miroir entre l'implicite et l'explicite, entre ce que l'auteur semble dire, veut dire et ce qu'il dit. Ainsi, parler gay a longtemps été un danger politique et social grave qui, à l'heure de la libération sexuelle, de la liberté d'expression, de la revendication de l'égalité entre les humains, échoue sur une plage de séquelles. Jean-Paul Aron avait la liberté politique et sociale de parler de sa condition, pourtant il semblerait à la lecture de son texte que le passé était trop lourd à porter et que les mots ne concordaient pas toujours entre son intention d'auteur et l'intentionalité de son texte. Il y a donc quelque chose de l'ordre d'une conscience marginale qui se considère toujours et encore comme fautive et cherche à se disculper de manière implicite.

Un autre facteur joue aussi dans la démarche de Jean-Paul Aron : la jalousie doublée du désir de réussite intellectuelle : "je me suis attaqué à lui par allergie philosophique, mais aussi pour une autre raison, à moitié illégitime : j'étais jaloux de sa gloire" (27). Jean-Paul Aron s'oppose donc existentiellement, dans la praxis, à Michel Foucault ; il se définit comme l'homme du vécu et du sens, et par conséquent contre la théorie et les structures de son ancien camarade. En rappelant la très grande gloire intellectuelle de Michel Foucault, il avoue aussi sa jalousie. Toutefois, cette expression "à moitié illégitime" vient encore ajouter une ambivalence, car il continue de voir sa démarche comme "à moitié légitime" : lui aussi aurait dû avoir droit à une plus grande reconnaissance sociale. Et d'une certaine manière, le texte de Jean-Paul Aron est une machine rhétorique qui lui permet de dépasser le maître sur un certain champ de vérité. Nous pourrions lire le texte de la manière suivante : Michel Foucault, le grand maître de ia théorie, du langage, du savoir et de la vérité, a menti en dissimulant sa condition ; moi, Jean-Paul Aron, l'homme du vécu et du sens, je dis toute la vérité. Je dépasse donc le maître dans la pratique.

Mon sida est un texte qui soulève plusieurs problématiques comme nous avons pu le voir. Maintenant, j'aimerais revenir plus précisément sur le rapport à l'implicite et à l'explicite. Soulignons que dans les Écrivains sacrifiés des années sida, Jean-Luc Maxence soutient que le thème du sida occupe une place privilégiée dans les derniers écrits de Michel Foucault, mais d'une manière implicite : 
le philosophe aurait écrit sur le sida de manière détournée, en omettant d'utiliser le mot directement. Dans ce cas, Michel Foucault n'aurait pas gardé un silence intellectuel absolu comme le prétend Jean-Paul Aron. Et ce dernier écrivait, en faisant référence au Pénis et la démoralisation de l'Occident : "Même si je ne m'y dévoilais pas et si le thème de l'homosexualité restait périphérique, il n'en restait pas moins bien présent" (16). Reconnaître la pratique d'une écriture gaie de l'implicite pour se disculper d'un très long silence, c'est, je le souhaite, reconnaître cette même pratique dans les oeuvres d'autres intellectuels gays. Il y aurait donc à mon avis cette dualité herméneutique de la révélation qui joue sur une poétique de l'implicite et de l'explicite dans l'écriture gaie et lesbienne. Le problème, c'est de théoriser une méthodologie qui permettra de déchiffrer cet implicite dans les différentes littératures (française, anglaise, espagnole, russe, japonaise, etc.), les différentes époques (de l'Antiquité gréco-romaine en passant par la tradition judéochrétienne et ce jusqu'à notre post-modernité) et les différentes sociétés (écrire gay en Amérique du Nord ne se fait pas de la même manière qu'en Afrique).

\section{Lisible, scriptible et recevable}

Comme nous l'avons vu au début de ma réflexion, il peut y avoir une censure imposée par le pouvoir politique et social interdisant de produire tout discours allant à l'encontre des règles établies, de l'hégémonie des valeurs choisies, de l'identité prônée pour chaque individu par le logocentrisme de ceux qui ont le droit de parler au nom de tous. Cette censure peut être jouée et déjouée à partir des marges, de ces lieux qui permettent d'attaquer le centre afin de le redéfinir : ce fut la stratégie des mouvements ethniques et féministes en occident. Mais pour cela il faut prendre sa liberté de parole en mains et être prêt à tout pour défendre son identité et sa vérité. Plusieurs n'ont pas le courage ou la force de le faire, alors ils préfèrent s'interdire de penser, de dire et d'écrire leur réalité. En évitant le blâme et la marginalisation, ils se condamnent au silence et à l'immobilité. En s'autocensurant ces êtres sont au monde sans exister ; fantômes sans vie ils vivent déjà la mort. Entre ces deux pôles, nous retrouvons des manifestations plus incertaines, plus difficiles à classer. C'est le cas de Mon sida de Jean-Paul Aron ; il 


\section{8 / Calderon}

prend la liberté de parler de son homosexualité et de sa maladie au moment où il n'a plus rien à perdre. L'auteur, conscient de sa situation sociale et des ressources à sa disposition, reconnaît que son comportement a été longtemps inconséquent : "mes réactions vis-àvis du sida jusqu'à ces dernières semaines, et ma gêne à me reconnaître tel que je suis, prouvent que j'ai été moi-même victime du cliché, du fantasme collectif face à une maladie innommable" (28). Comme je l'ai montré auparavant, le discours de Jean-Paul Aron qui se veut le témoignage de son vécu à travers un langage de vérité, oscille entre ce que l'auteur voudrait dire et ce qu'il se permet de dire, entre ce qu'il communique et ce qu'il voudrait communiquer. Il n'y a pas d'adéquation entre l'intention de l'auteur et l'intentionalité du texte, parce que ce qui est dit de manière explicite renvoie immédiatement à un sens implicite qui révèle une toute autre interprétation du texte. Et, malgré le fait que Jean-Paul Aron essaie de désamorcer les préjugés sociaux face à l'homosexualité et au sida, ses propres idées et valeurs sont en contradiction avec ce qu'il voudrait dire. Parfois il fait l'illustration et la défense de l'homosexualité, et d'autres fois une toute autre image de ce phénomène apparaît au fil du discours. Il écrit : "L'homosexualité est une forme de déviation, de marginalité que le corps social peut supporter sans l'avaliser jusqu'au bout. Le sida réintroduit la condamnation" (30). Ceci m'amène à souligner que Jean-Paul Aron est un homme d'avant la Révolution sexuelle des années 70 qui aimerait tenir à 62 ans (en 1987) les propos d'un gay complètement émancipé ; malheureusement ce n'est pas ce qui se produit. Nous sentons tout au long de son plaidoyer une profonde influence de la culture judéo-chrétienne occidentale. Rappelons simplement le paradigme de la confession, de la faute, de la Loi et de la condamnation qui se dégage du texte en général et du passage que je viens de citer en particulier.

Finalement ce qui a lieu dans ce texte peut être expliqué par les notions de lisibilité, de scriptibilité et de recevabilité développées par Roland Barthes. Ce qui est lisible dans Mon sida de Jean-Paul Aron, c'est le niveau textuel de ce qui a déjà été écrit par d'autres et que n'importe qui peut réécrire sans aucune innovation ; c'est tout ce qui dans les propos de l'auteur est explicite. Tout lecteur comprend immédiatement l'appel à l'ouverture d'esprit, à 
l'acceptation et à l'aide des malades. Ce qui est scriptible, c'est ce que je perçois dans le texte mais en le lisant avec peine. Je dois changer un peu mon régime de lecture pour comprendre ce qui se passe entre ce que le texte communique de manière explicite et ce qu'il veut dire implicitement. Dans Mon sida, ce phénomène touche le système de la signification entre les notions de justification, de culpabilité, d'homosexualité, de sida, d'identité, d'appartenance, d'épanouissement, etc., idées extrêmement problématiques pour l'auteur. Et puis, il y aurait un autre lieu du sens, où je peux recevoir le texte sans tout à fait comprendre ce qui se passe, sans saisir ce que ce texte apporte d'inouï. Roland Barthes expliquait ce phénomène par la remarque suivante : "je ne puis lire ni écrire ce que vous produisez, mais je le reçois, comme un feu, une drogue, une désorganisation énigmatique" (109). C'est encore un peu cela le texte gay et lesbien. Nous n'avons qu'à penser à l'écriture d'Hervé Guibert : son oeuvre est recevable, mais sur bien des côtés illisibles et inescriptible - ce fut le cas aussi pour toute la production du Nouveau Roman, il n'y a pas si longtemps -, de là tout son intérêt et l'importance de continuer à réfléchir sur la littérature gaie et lesbienne.

\section{Ouvrages cités}

Aron, Jean-Paul. Mon sida. Paris : Christian Bourgois Éditeur, 1988. Bakhtine, Mikhaïl. La Poétique de Dostoïevski. Traduit du russe pas Isabelle Kolitcheff. Paris : Seuil, 1970.

Barthes, Roland. Roland Barthes par Roland Barthes. Paris : Seuil, 1995.

Guibert, Hervé. À l'ami qui ne m'a pas sauvé la vie. Paris : Gallimard, 1990.

Maxence, Jean-Luc. Les écrivains sacrifiés des années sida. Paris : Bayard, 1995.

Pollak, Michael. Les homosexuels et le sida : sociologie d'une épidémie. Paris : Métailié, 1988. 